\title{
DREDGING TECHNIQUE FOR KERATOCYSTIC ODONTOGENIC TUMOR: A FIVE-YEAR FOLLOW-UP OF 28 CASES
}

\author{
Waleed R. El-Beialy,*** and Nadia Galal
}

\begin{abstract}
Keratocystic Odontogenic Tumor (KCOT) is a locally aggressive tumor with high recurrence rate, and has a distinct and characteristic histologic appearance contributing to such aggressiveness and recurrence. Such wild nature of this tumor had led to even more aggressive treatment modalities in the past. Better understanding of the nature of this tumor allowed for simpler approaches including enucleation with or without adjuvant therapy, and decompression with or without residual cystectomy. Dredging technique is one of the least invasive treatment modalities suggested for the management of KCOT. In this study following our preliminary reported outcomes, we here within report a five-year follow-up of 28 mandibular KCOT cases (age range 14-43 years; mean 28.5years) with ZERO recurrence.
\end{abstract}

KEYWORDS: KOTs, KCOTS, Keratocystic odentogenic tumor , Dredging technique

\section{INTRODUCTION}

Keratocystic Odontogenic Tumor (KCOT) was thought to be an epithelial origin developmental cyst of the jaws first reported in 1956 and formerly known as the odontogenic keratocyst (OKC). Its locally aggressive behavior, high recurrence rate, distinct and characteristic histologic appearance caused the World Health Organization (WHO) in 2005 to reclassify this lesion as a tumor instead of a cyst. ${ }^{(1,2)}$ KCOT is one of the most aggressive odontogenic begnin tumors of the oral cavity. KCOT is known for its rapid growth and its tendency to invade the adjacent tissues including bone. KCOTs are commonly seen in the mandible with the majority occurring in the angle of the mandible and ramus. ${ }^{(1,3)}$ Radiographically, displacement of impacted or erupted teeth, root resorption, root displacement, or extrusion of erupted teeth may be evident. ${ }^{(1)}$ KOTs may occur in any part of the jaws; however, they are commonly encountered with ameloblastomas, calcifying epithelial odontogenic tumors, and myxomas. KCOT can be classified on the basis of locularity namely unilocular and multilocular. Unilocular is isolated but not

* Associate Professor, Oral \& Maxillofacial Surgery Department, Faculty of Dentistry, Cairo University ** Associate Professor, Oral \& Maxillofacial Surgery Department, Faculty of Dentistry, Future University in Egypt 
necessarily; while multilocular is mostly associated with Naevus Basal Cell Carcinoma syndrome(Gorlin Syndrome). It has a predilection for the posterior body of the mandible and ascending ramus. ${ }^{(4)}$ KOTs are of great interest among oral and maxillofacial surgeons because of their high recurrence rate. Recent literature has reported a recurrence rate of $0 \%-50 \% .{ }^{(5)}$ The real reasons for the high recurrence rate of KCOTs remain substantially unknown. ${ }^{(6)}$

There is no consensus over the most appropriate treatment modality for this tumour. The common strategies include marsupialization and enucleation, which may be combined with adjuvant treatments such as application of Carnoy's solution, cryotherapy, peripheral ostectomy, or even marginal or radical resection. All of which target reduction of recurrence rate with the minimal intervention. ${ }^{(6,7)}$

As previously described in our preliminary study ${ }^{(6)}$ Dredging Technique has been employed to eradicate KCOT and restore the normal contour and function of jaw with a minimalistic approach. "Dredging Method" is a conservative surgical procedure in which, after deflation and enucleation or only enucleation, repeated dredging is applied to accelerate new bone formation by removing out the scar tissue from the bony cavity. ${ }^{(6,8.9)}$

\section{PATIENTS AND METHODS}

A total of 28 patients (22 males $79 \%$ and 6 females $21 \%$ ) suffering from large KCOT more than $3 \mathrm{~cm}$ in its largest diameter (2 maxillary KCOT and 26 mandibular KCOT) were included in this study. Patients' age ranged from 14 to 43 years (mean 28.5 years). Treatment time for full resolution and closure varied from 3 months to 1.8 years. The diagnosis of each patient was performed through clinical and radiographic examination, aspiration and incisional biopsies. Complete medical questionnaires were obtained from each patient to evaluate their past and present medical status and to reveal any existing problems, which might need further investigations and consultation, such as Gorlin Syndrome.

All patients included in the study were informed about the high recurrence rate of their lesions and the alternative lines of treatment possible (as resections of the jaw) versus Dredging technique and the prognosis of each. The patients who agreed to perform dredging technique were included in the study and signed a written consent to perform a minimum of a yearly radiographic follow up for up to 10 years post operatively. The operation was performed under local anesthesia for most patients (25 cases) and under general anesthesia (GA) using naso-endotracheal intubation (3 cases, based on their request).

All patients were followed up both clinically and radiographically for a minimum of 5-years postoperatively.

"Dredging Method" is a conservative surgical procedure in which, deflation is carried out through marsupilization and open packing of the lesion using antibiotic treated vaslinized gauze. Marsupilization causes deflation of the lesion, where portion of the cystic wall, overlying bone and mucoperiosteum are removed in order to release intracystic pressure and facilitate the formation of a clear bony outline. $(8,9)$ The drain is frequently changed and reduced in size to avoid pressure that might prevent bone formation, with intermittent irrigation using Betadine (Povidone-iodine) and hydrogen peroxide. Enucleation is only performed following cessation of bone formation that is identified by formation of clear bony outline. After enucleation the KCOT is removed completely along with a portion of surrounding healthy bone, and then the bony cavity is still packed open. Repeated dredging out 
of the scar tissue that fills up the bony cavity and prevents the bone formation is commenced as many times as necessary. Enucleation is applied in 2-3 months interval to accelerate new bone formation and eliminate any residual tumor. Histological examinations for all specimens throughout the repeated dredging, are mandatory to ensure elimination of residual tumor cells and prevent recurrence. In "Dredging Method" the follow up begins when the tumor cells are not identified in microscopic examinations of the scar tissues removed by 2 consecutive dredging. Continuous and regular follow up is an essential part of the treatment. Patients were routinely covered with broad-spectrum antibiotics (usually penicillin and for allergic patients: cephalosporin), and NSAIDS for the first postoperative week.

\section{RESULTS}

In the current study, 36 cases that have been histologically confirmed as KCOT were treated using Dredging technique. All patients are still being followed up, at least on a yearly basis for those who had full resolution. Only 28 patients completed the minimum follow-up interval, which was 5 years for all 28 patients; demographic data demonstrated in (Figure 1). None of the patients experienced recurrence of the primary KCOT lesion, up to five years postoperatively, neither histologically during dredging stages nor radiographically following full resolution. (Figure 2 \& 3) All initial dredging biopsies demonstrated the histological pattern of KCOT that transformed into cystic outline (loss of KCOT nature) highly infiltrated by chronic inflammatory cells in later biopsies. Three cases exhibited remnant KCOT along with inflamed cystic outline in the second dredging biopsy; and one case in the third dredging biopsy; however, further dredging biopsies for these cases demonstrated inflamed cystic lining with loss of KCOT characteristics. Three cases experienced premature closure after first time dredging due to lack of initial follow up and only two cases needed repetition of stage one dredging. All patients learned how to change their drains and properly irrigate the surgical site. In the final stages, there was no need for a drain as the defect was very shallow.

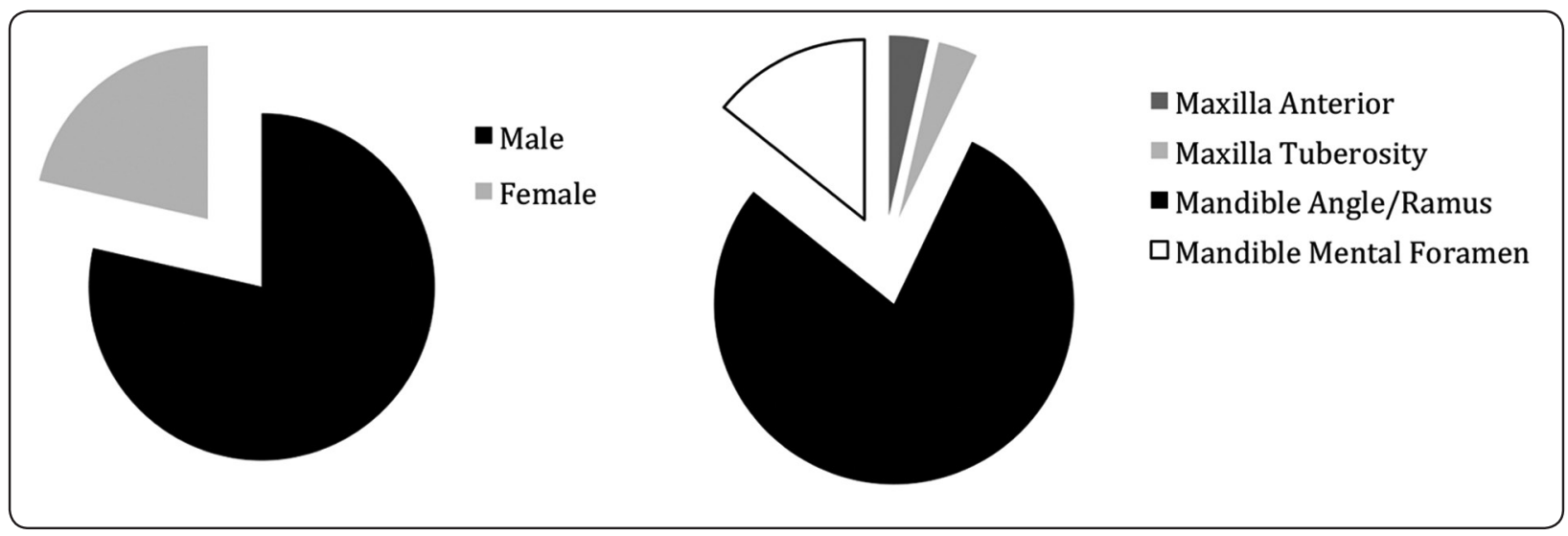

Fig. (1) Demographic data displayed as a pie chart. (A) Male (22; 79\%) to Female ratio (6:21\%). (B) Anatomical location: Anterior Maxilla (1; 4\%); Tuberosity (1; 4\%); Angle/Ramus (22; 78\%) and Related to Mental foramen (4; 14\%). 

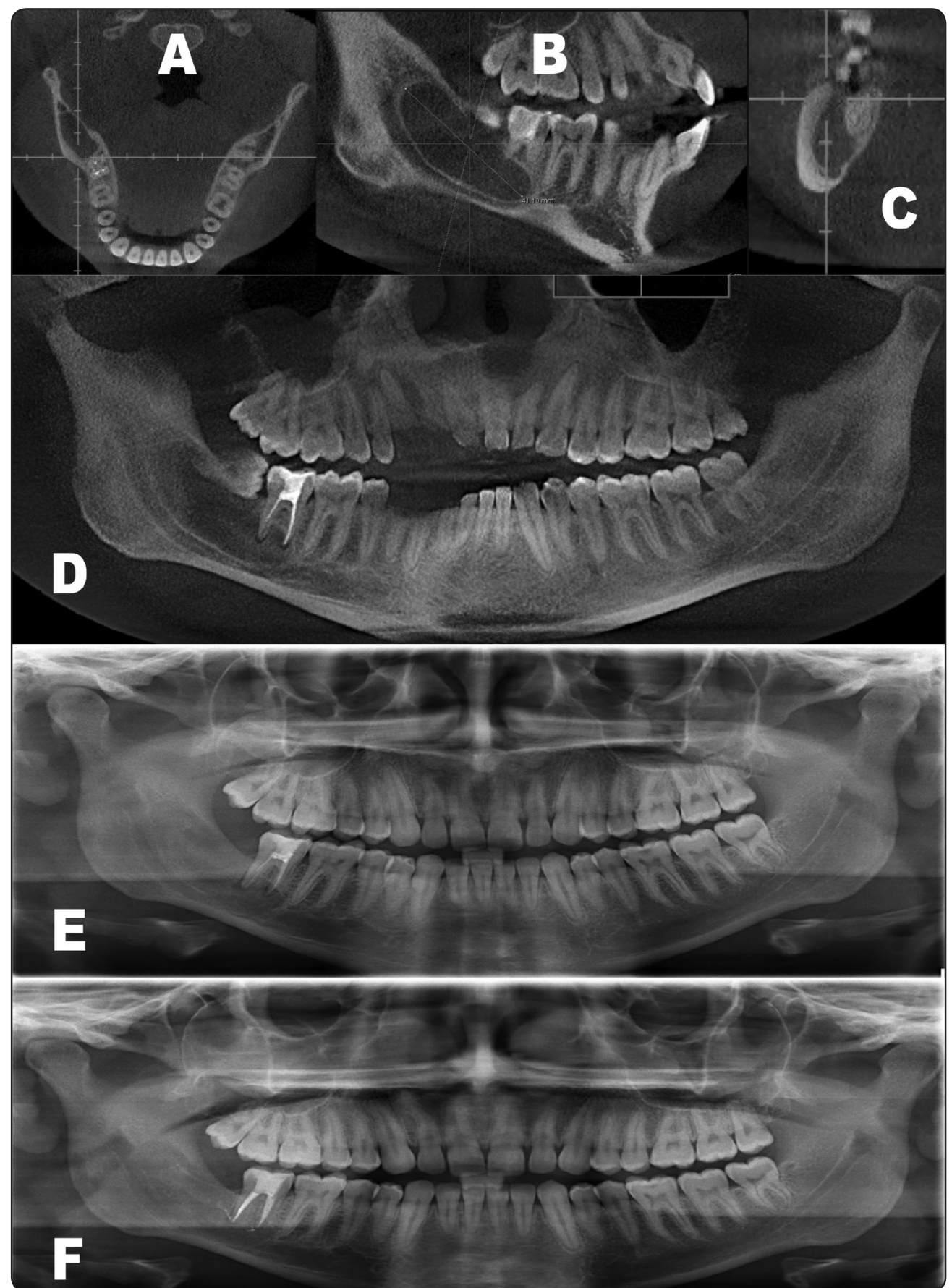

Fig. (2) A 23 year KCOT old case radiographic follow-up, demonstrating the gradual new bone formation and reduction in KCOT size at: A-D) Before treatment KCOT related to an impacted mandibular third molar; A) Axial Cone Beam Computerized Tomogram (CBCT) cut; B) Lateral (Sagittal) CBCT cut, demonstrating an KCOT of $41.3 \mathrm{~mm}$ at its widest diameter; C) Coronal (Facial) CBCT cut demonstrating involvement of the inferior alveolar nerve; D) Preoperative orthopantograph. E) Mid postoperative orthopantograph; F) End of treatment orthopantograph. 


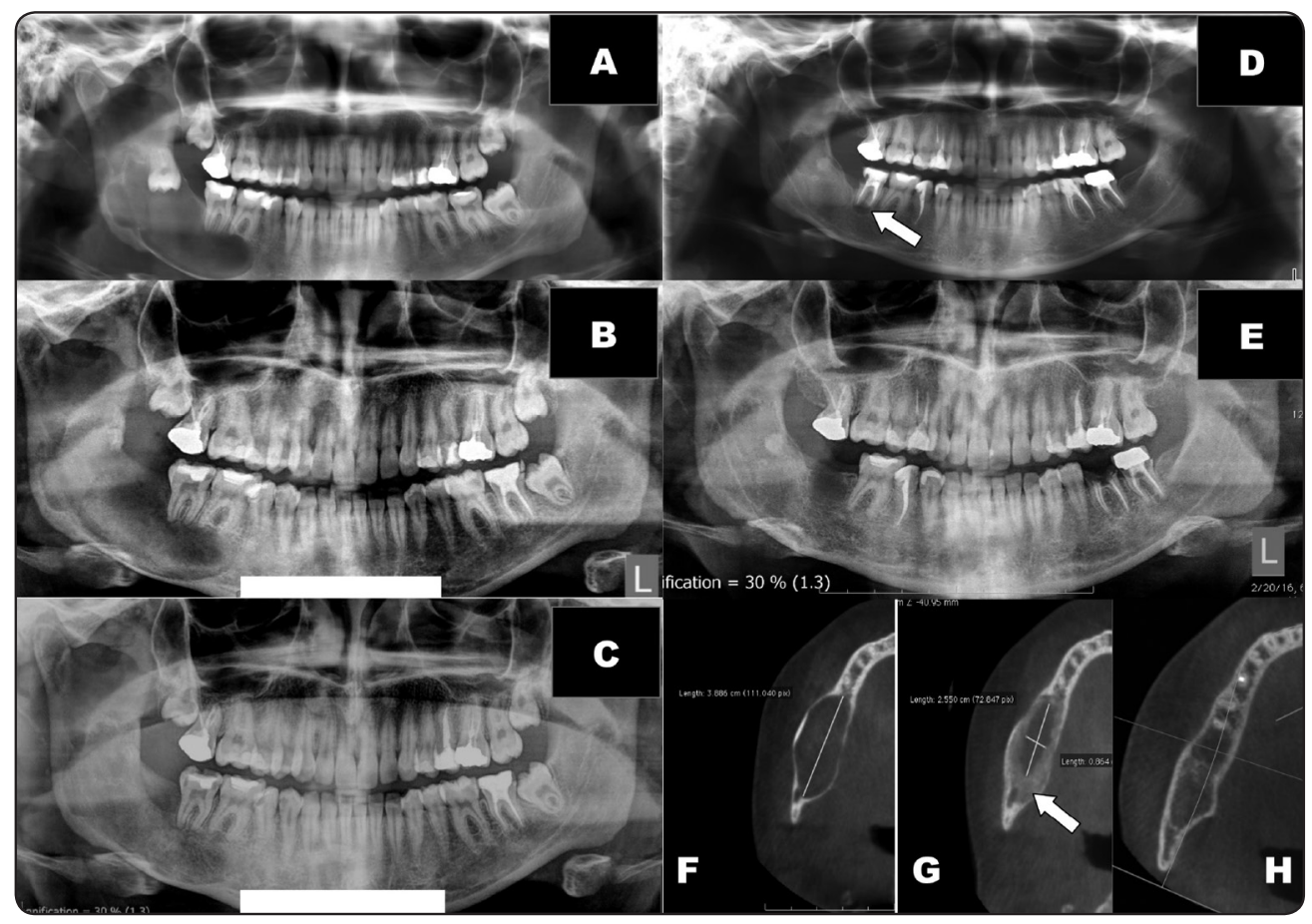

Fig. (3) A 19 year old case follow-up using orthopantogramic radiographs, demonstrating the gradual new bone formation and reduction in KCOT size at A) Before treatment KCOT related to an impacted mandibular third molar $(38.8 \mathrm{~mm}), \mathrm{B})$ Following first stage of Dredging technique and extraction of the impacted mandibular third molar (25.5mm); C) The KCOT site filled with new bone formation. D) 5-years follow-up showing periapical radiolucency related to endodontic treated mandibular right second molar (white arrow). (E) Following persistence of radiolucency despite endodontic retreatment and failure of attempts to salvage the tooth. Surgical extraction of the affected tooth along with enucleation of the periapical tissues and histopathological staining that turned out to be chronically infiltrated granulation tissue (7-years and 1 month postoperatively); Axial CBCT cuts (F) Preoperatively; (G) Mid treatment, (white arrow pointing circumferential new bone formation; and (H) 7-years 1 month following extraction of mandibular second molar and enucleation of histopathologically confirmed periapical granuloma.

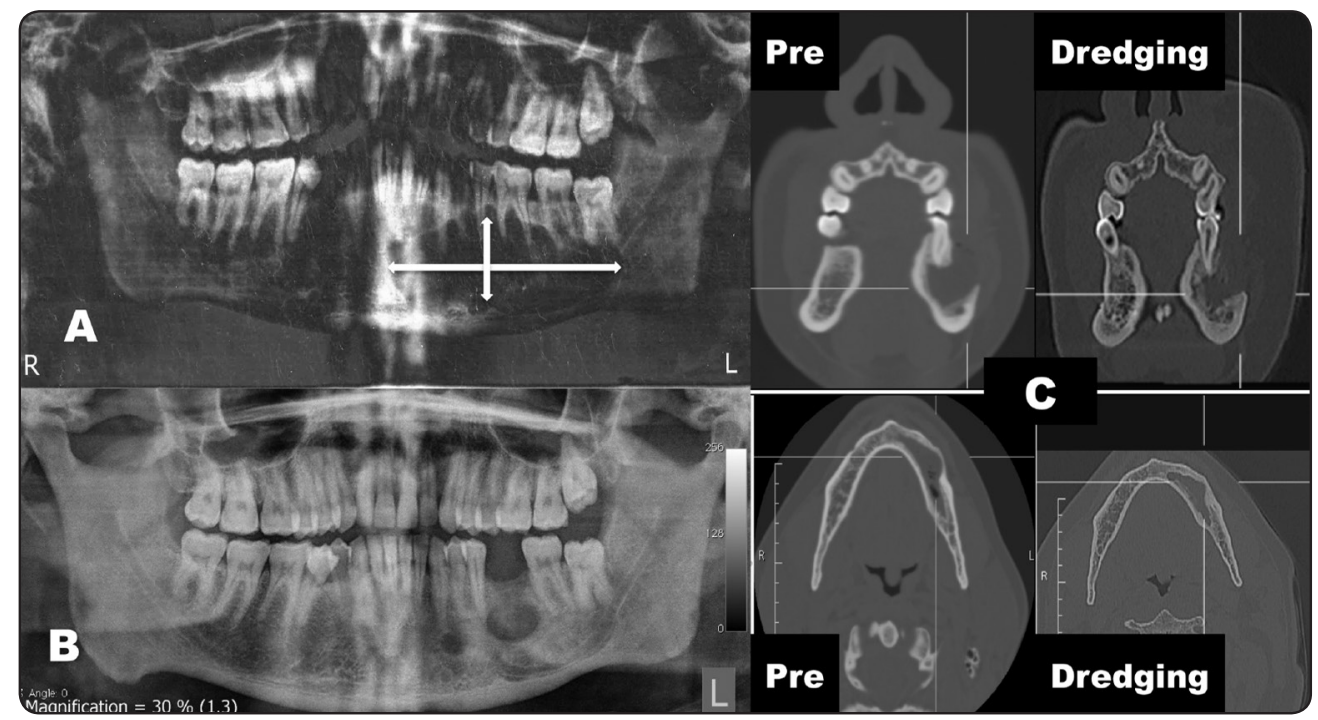

Fig. (4) Orthopatograph (left) and CT cuts (right) of a 40 year old patient. (A) Preoperatively; (B) Postoperatively, the residual radiolucency is a buccal defect in cortical bone only; (C) CT cuts: preoperatively (Left panel) and during Dredging technique (Right panel): demonstrating new bone formation and reduction in the size of the defect. 


\section{DISCUSSION}

The treatment of KCOT remains controversial, and there is no consensus as to whether an aggressive treatment is superior to conservative treatment in reducing the recurrence rate for KCOT patients. ${ }^{(7,10)}$ Treatment targets are to remove the lesion entirely, to avoid complications and recurrence. While resection is warranted for highly recurrent lesions, yet the residual deformity necessitates reconstruction either immediate or delayed. However, immediate reconstruction still caries the risk of recurrence. Moreover, KCOT is still a benign tumor and should be treated as one. Moreover, facial and skeletal contour is critically influential on the function and facial aesthetics. Also, any treatment modality for such a condition becomes inadequate if it causes any sort of intra- or extra-oral deformity. Deformity of the oral cavity causes functional inconvenience, aesthetic dissatisfaction and mental agony. So, the purpose should be correction of the disorder as well as restoration of normal contour and function of jaw. ${ }^{(9,11)}$ Dredging technique as presented in the current study is considered fulfilling to these purposes, especially in benign lesions as KCOT. It is seen that after deflation and enucleation, the tumor cells are identified in the scar tissue within the bony cavity, which is the cause of recurrence. ${ }^{(6)}$ So the scar tissue should be dredged out repeatedly to prevent the recurrence and to accelerate new bone formation. No recurrence by this technique was encountered similar to other reports. ${ }^{(12)}$ As we previously reported following cessation of identification of tumor cells in histologically examined biopsies the dredging is continued only for restoration of bony defect. ${ }^{(6)}$

Considerations should be given to the age of patient, site, nature, extension of lesion. It is recommended that this technique should not be applied if the patient is not totally motivated for long term duration of follow up, and fully understands the possibility of recurrence as indicated in his/her consent. Failing to adhere to follow up instructions, as was the situation with three cases in the current study, who failed to adhere to the initial strict follow-up following the initial step of dredging, due to different reasons. This led to loss of the drain and closure of the dredged site in two cases that had to be repeated.

In the current study, following aspiration biopsy, the first stage of dredging was commenced and the retrieved tissue was histopathologically stained and examined as an incisional biopsy. It is our experience that this was a better choice for the patient and time saving procedure rather than performing an incisional biopsy as a separate step prior to treatment. Biopsies are the corner stone for a definitive treatment plan, despite the fact that overly destructive lesions and those infiltrating into surrounding soft tissues and deeper structures are generally dictative of resections despite their nature. Conservative versus aggressive approaches remains debatable throughout the literature, particularly for less well-understood lesions such as KCOT. Significantly, studies have shown that the orthokeratinized KCOT had a substantially lower recurrence rate than those that were parakeratinized, and later molecular studies showed significant differences between the two varieties. ${ }^{(10)}$ Unfortunately, there is not yet clarity regarding the behavior of KCOT that show both orthokeratotic and parakeratotic areas histologically. ${ }^{(13)}$ No significant histologic variation was seen post decompression in our series of patients.

More aggressive approaches recommended in literature include removal of the whole lesion and further treating its lumen Carnoy's solution before or after removal, and curettage of the bony wall, or even more aggressive approaches as radical resection. ${ }^{(14,15,16)}$ Worthy of note, the destructive effect of such chemical cauterization on the exposed inferior alveolar neurovascular bundle. ${ }^{(7,17)}$ As encountered in the current study were most cases had exposed inferior alveolar nerves within the lesion 
or KCOT were encapsulating the mental nerve, which prevented the use of such chemocauterizing agent. Moreover, all of our patients retained full sensation of the mental nerve with no anesthesia or parastheisa; which is one of the unique advantages of Dredging technique. Of note, the fact that 5 of the patients were dentists, who preferred Dredging over resection and accompanied severing of the inferior alveolar nerve, reconstruction and implants. Perhaps the most extensive form of treatment indicated for the management of select KCOT's is that of osseous resection (marginal or segmental). Although some authors consider this treatment to be radical for a benign tumor, en bloc resection is the only treatment modality consistently cited in case series with a zero recurrence rate. Hence, some considers it the preferred approach for a recurrent lesion. ${ }^{(15)}$ Of academic concern is the lack of consensus concerning the margins required in a primary resection to ensure removal of all possible satellite cysts. Anecdotal reports have suggested that a minimum $5 \mathrm{~mm}$ bony margin is adequate to ensure satellite cyst removal. However, as evident throughout the literature it is seldom to find such a safety margin to perform en bloc resections. Dredging technique needs to be popularizes as a possible modality of treatment for patients who are taught about its benefits and limitations, further studies with larger patient samples are recommended. Resection is a form of amputation and should be reserved for malignant lesions.

\section{CONCLUSIONS \& RECOMMENDATIONS}

Dredging technique is a note worthy treatment modality for KCOT. Despite its simplicity paves the way for a simpler approach to managing KCOT. It eliminates the need for resections and associated consequences which include but are not limited to: loss of mandibular integrity, nerve amputation, consequent loss of sensation, need for reconstruction (remote donor site, donor site morbidity...etc.), complicated and delayed restoration of occlusion and function. Patient motivation and cooperation is a crucial factor in the success of treatment. Considerations should be given to the age of patient, site, nature, extension of lesion. Younger patients seem to reach resolution much faster than older ones. Residual bony walls are a crucial factor for the success of Dredging, regardless of the thickness of such bone.

\section{Limitations}

This is a 5-year prospective study on a limited number of patients (28 patients), this should be taken into consideration when analyzing the results. Further research with longer follow-up intervals and on a larger sample of patients should are advisable.

\section{Ethical approval}

The work not required approved by the appropriate ethical committees related to the institutions in which it was performed, as it is a welldocumented technique and previous pilot study (6) was approved by the ethical committee.

\section{REFERENCES}

1. Brannon R. The odontogenic keratocyst. A clinicopathological study of 312 cases. Part I. Clinical features. Oral Surg Oral Med Oral Pathol Oral Radiol Endod. 1976; 42: p. 54-72.

2. Barnes S, Eveson J, Reichart P, Sidransky D. World Health Organization classification of tumours. Pathology \& genetics of Head and neck tumours. ; 2005.

3. Browne R. The odontogenic keratocyst: clinical aspects. Br Dent J. 1970; 128: p. 225-231.

4. Myoung H, Hong S, Hong S, Lee J, Lim C, Choung P. Odontogenic keratocyst: review of 256 cases for recurrence and clinicopathologic parameters. Oral Surg Oral Med Oral Pathol Oral Radiol Endod. 2001; 91(3): p. 328-333.

5. Johnsons N, Batstone M, Savage N. Management and recurrence of keratocystic odontogenic tumor: a systematic review. Oral Surg Oral Med Oral Pathol Oral Radiol. 2013; 116(4): p. 271-276. 
6. Galal N. Managment of Keratocystic Odontogenic Tumor by DREDGING Technique. Egyptian Dental Journal. 2015 April; 61: p. 1-7.

7. Al-Moraissi E, Pogel MA, Ellis III E. Enucleation with or without adjuvant therapy versus marsupialization with or without secondary enucleation in the treatment of keratocystic odontogenic tumors: A systematic review and metaanalysis. Journal of Cranio-Maxillo-Facial Surgery. 2016;: p. 1-9.

8. Kawamura M. A proposal for the treatment of ameloblastoma. 29:765. Jpn. J. Oral Maxillofac. Surg. 1983; 29: p. 765 .

9. Kawamura M. KI,IN. Dredging method-a new conservative treatment approach for cystic lesions and tumors of the jaw.. Hokkaido J. Dent. Sci. 1988; 9 : p. 104-106.

10. Wright J. The Odontogenic Keratocyst: Orthokeratinized variant. Oral Surg Oral Med Oral Pathol. 1981; 51(6): p. 609-618.

11. Kobayashi I, Kawamura M, Amemiya A, al e. Histopathological pattern of ameloblastoma and choice of treatment method. J. Jpn. Soc. Oral Tumor. J. Jpn. Soc. Oral Tumor. 1985; 3: p. 45-48.
12. Kawamura M, Inoue N, Kobayashi I, Ahmed M. "Dredging Method"-a New Approach for the Treatment of Ameloblastoma. Asian J. Oral Maxillofac. Surg. 1991; 3: p. 81-88.

13. Morgan T, Burton C, Qian F. A retrospective review of treatment of the odontogenic keratocyst. J oral Maxillofac Surg. 2005; 63(5): p. 635-633.

14. Williams T, Connor FJ. Surgical management of the Odontogenic Keratocyst: AggressiveAproach. J oral Maxillofac Surg. 1994; 52(9): p. 964-966.

15. Enisidis G, Fock N, Sulzbacher I, Ewers R. Conservative treatment of large cystic lesions of the mandible: a prospective study of the effect of decompression. Br J Oral Maxillofac Surg. 2004; 42(6): p. 546-550.

16. Kolokythas A, Fernandes R, Pazoki A, Ord R. To Decompress or Not to Decompress? A Comparative Study between Decompression and Enucleation Vs. Resection/ Peripheral Ostectomy. J oral Maxillofac Surg. 2007; 65(4): p. 640-644.

17. Meiselman F. Surgical management of the Odontogenic Keratocyst: Conservetive Aproach. J Oral Maxillofac Surg. 1994; 52(9): p. 960-963. 